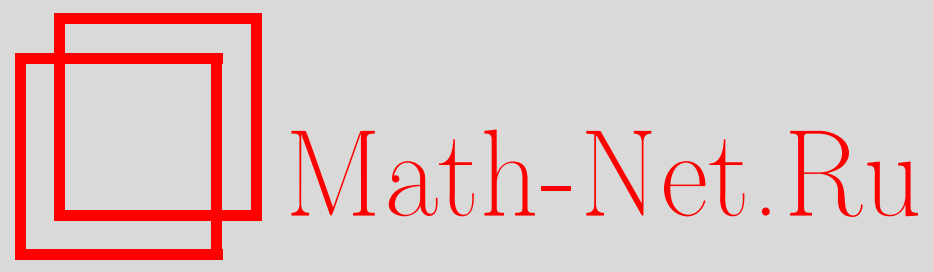

Н. Л. Чуприков, Об эффекте Хартмана и скорости распространения электромагнитной волны в процессе туннелирования, Вестн. Сам. гос. техн. ун-та. Сер. Физ.-мат. науки, 2013, выпуск 2(), 215-222

DOI: https://doi.org/10.14498/vsgtu1147

Использование Общероссийского математического портала Math-Net.Ru подразумевает, что вы прочитали и согласны с пользовательским соглашением

http://www.mathnet.ru/rus/agreement

Параметры загрузки:

IP : 54.237 .59 .107

26 апреля 2023 г., $17: 57: 01$

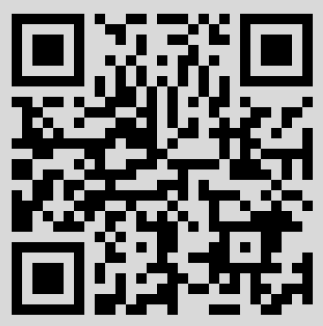


Вестн. Сам. гос. техн. ун-та. Сер. Физ.-мат. науки. 2013. № 2 (31). C. $215-222$

УДК 537.87; 621.371; 517.958:537.8; 517.958:535

\title{
ОБ ЭФФЕКТЕ ХАРТМАНА И СКОРОСТИ РАСПРОСТРАНЕНИЯ ЭЛЕКТРОМАГНИТНОЙ ВОЛНЫ В ПРОЦЕССЕ ТУННЕЛИРОВАНИЯ
}

\author{
Н. Л. Чуприков \\ Томский государственный педагогический университет, \\ Россия, 634061, Томск, ул. Киевская, 60. \\ E-mail: chnl@tspu.edu.ru
}

Представлен новый подход $\kappa$ задаче рассеяния плоской электромагнитной ТЕ волны на однородном слое диэлектрика, которая, в отличие от стандартной модели, не предсказывает эбфект Хартмана для времени туннелирования в случае рассеяния ТЕ волны в режиме нарушенного полного внутреннего отражения (НПВО). Основная идея подхода состоит в том, что корректное определение скорости и времени туннелирования возможно лишь в том случае, если динамика обоих подпроцессов - прохождения и отражения - известна на всех этапах исследуемого прочесса рассеяния. Показано, что вигнеровское (групповое) время туннелирования введено без учета этого требования и, как следствие, ни сама характеристика, ни связанный с нею эбфект Хартмана не имеют никакого отношения $\kappa$ переносу энергии волны через слой. Время туннелирования как время пребывания имеет $к$ этому прямое отношение, и оно не приводит $\kappa$ эфбекту Хартмана.

Ключевые слова: групповая скорость, энергетическая скорость, время туннелирования, прохождение, отражение.

Введение. Как известно [1], в квантовой механике при анализе времени туннелирования в рамках концепции групповой скорости волнового пакета был обнаружен эффект Хартмана, суть которого состоит в том, что вигнеровское (групповое) время туннелирования как функция ширины потенциального барьера, через который туннелирует частица, выходит на насыщение при неограниченном росте этого параметра. Позднее этот эффект был обнаружен и в электродинамике для плоской монохроматической световой волны, рассеивающейся на воздушном зазоре между стеклянными призмами в режиме НПВО (см. [2]). Таким образом, в существующих стандартных моделях туннелирования концепция групповой скорости приводит к скоростям туннелирования, превышающим $c$ - скорость света в вакууме.

Несмотря на необычность этого результата с точки зрения специальной теории относительности (СТО), в классической электродинамике эффект Хартмана получил в настоящее время статус экспериментально наблюдаемого эффекта (см., например, [3-7]), который нужно лишь «примирить» с принципами СТО. Впрочем, и в этом вопросе, за исключением отдельных авторов (см., например, [3]), имеется практически полное единодушие - принято считать, что возникновение сверхсветовых групповых скоростей в задаче туннелирования не противоречит СТО.

В пользу этого обычно приводят два аргумента (см., например, [7]). Один из них состоит в том, что запрет на сверхсветовые скорости в СТО касается только скорости переноса информаиии. Что ни (бесконечная) монохроматическая световая волна, характеризуемая энергетической скоростью, ни

Николай Леонидович Чуприков (д.ф.-м.н., доц.), профессор, каф. теоретической физики. 
(локализованный) волновой пакет с гладкой огибающей, характеризуемый групповой скоростью, не переносят информацию. Что о переносе информации можно говорить лишь в том случае, если огибающая волнового пакета имеет разрывы и скорость перемещения переднего разрыва (фронта) волнового пакета как раз и есть скорость переноса информации - сигнальная (или фронтальная [7]) скорость. То есть СТО налагает ограничение сверху только на сигнальную скорость света в среде. Другой аргумент состоит в том, что если диэлектрические проницаемости всех сред в слоистой структуре, в которой распространяется световой импульс, удовлетворяют дисперсионным соотношениям, то выполнение принципа причинности при описании (под)процесса туннелирования гарантируется автоматически. Однако ни один из этих аргументов не выдерживает критики.

Во-первых, СТО - это физическая теория, и поэтому все ее положения распространяются на физические объекты, то есть, на те, чьё движение связано с переносом вещества и энергии и подчиняется физическим законам. Это или частицы, или поля. Нет физических законов, сформулированных для «информации» как таковой. Само понятие информации не имеет четкого определения в физике. В частности, это выражается в том, что сигнальная скорость, призванная характеризовать скорость движения светового импульса, описывает, строго говоря, лишь бесконечно малую часть этого импульса, примыкающую непосредственно к его переднему фронту (разрыву), и, следовательно, эта величина не содержит в себе никакой информации о динамике основной части импульса. Слабость этой концепции по сравнению с групповой и энергетической скоростями видна уже из того, что групповая скорость, хотя и относится тоже только к одной точке волнового пакета - к его «центру масс» (ЦМ), все же является интегральной характеристикой пакета. Что касается энергетической скорости, ее достоинство состоит в том, что она определяется для каждой точки пакета, а не для одной, как сигнальная скорость.

Во-вторых, прежде чем утверждать, что сверхсветовые групповые скорости туннелирования заведомо не нарушают принципа причинности, необходимо знать динамику этого (под)процесса на всех этапах рассеяния. Однако в существующих подходах это требование не выполнено. Если на конечной стадии рассеяния в этих моделях фигурируют два волновых пакета - прошедший и отраженный, то на его первой стадии - только один, падающий волновой пакет. При этом между падающим и прошедшим волновыми пакетами нет причинно-следственной связи [8] - ни передний фронт, ни ЦМ падающего волнового пакета не преобразуются, соответственно, в передний фронт и ЦМ прошедшего пакета.

При определении времени туннелирования в стандартном подходе этот факт просто игнорируется. Это проявляется в том, что групповое время задержки для туннелирования вводится как разность времени прибытия ЦМ прошедшего волнового пакета в точку, достаточно удаленную от слоя (или потенциального барьера) и времени прибытия ЦМ «опорного» волнового пакета, который совпадает в начальный момент времени с падающим волновым пакетом и движется свободно во все моменты времени. То есть стандартный подход базируется на неявном предположении, что прошедший волновой пакет связан каузально с падающим волновым пакетом. Но это не соответствует действительности [8]. Так что групповое время задержки и связанное с ним вигнеровское время туннелирования определены изначально с нарушением принципа причинности. И то, что аномально короткое и даже отрицательное 
вигнеровское время туннелирования наблюдается экспериментально, говорит лишь о том, что и эксперимент, и тестируемая концепция времени туннелирования базируются на одной и той же, изначально нарушающей принцип причинности, процедуре хронометрирования.

Итак, корректное определение скорости и времени туннелирования предполагает раздельное описание динамики прошедшего и отраженного волновых пакетов на всех этапах рассеяния. Для квантового процесса туннелирования такая модель была предложена в [9] (см. также $[10,11])$. В данной работе мы распространяем этот подход на аналогичную задачу рассеяния (см., например, [12]) в классической электродинамике.

1. Постановка задачи. Рассмотрим две однородные немагнитные $(\mu=1)$ среды с диэлектрическими проницаемостями $\epsilon_{0}$ и $\epsilon$ : среда с коэффициентом преломления $n(n=\sqrt{\epsilon})$ заполняет интервал $[0, d]$ на оси $O Z$, а «фоновая» среда с коэффициентом преломления $n_{0}\left(n_{0}=\sqrt{\epsilon_{0}}\right)$ заполняет пространственные области, лежащие по обе стороны от этого интервала; $n, n_{0} \geqslant 1 ; n \neq n_{0}$. Предполагаются, что в обеих средах нет ни затухания, ни дисперсии.

Пусть плоская световая ТЕ волна падает слева на границу $z=0$ при условии, что ее волновой вектор лежит в плоскости $Y Z$, а угол между этим вектором и осью $O Z$ равен $\theta$. В этом случае только одна компонента, $E_{x}$, электрического поля и две компоненты, $H_{y}$ и $H_{z}$, магнитного поля отличны от нуля. Чтобы воспользоваться аналогией между квантовой и оптической задачами туннелирования, будем искать эти величины в комплексной форме.

Поскольку исследуемая структура неоднородна только в $z$-направлении,

$$
\begin{gathered}
E_{x}=U(z) e^{i \chi}, \quad H_{y}=V(z) e^{i \chi}, \quad H_{z}=W(z) e^{i \chi} \\
\chi=k n_{0, y} y-\omega t, \quad n_{0, y}=n_{0} \sin \theta, \quad k=\omega / c
\end{gathered}
$$

Тогда искомые вещественные проекции $-\operatorname{Re}\left(U e^{i \chi}\right), \operatorname{Re}\left(V e^{i \chi}\right)$ и $\operatorname{Re}\left(W e^{i \chi}\right)$. Для плотности энергии $w$ и вектора Умова-Пойнтинга $\mathbf{S}$ внутри слоя имеем

$$
\begin{gathered}
w=w^{(0)}+w^{(t)}, \quad \mathbf{S}=\mathbf{S}^{(0)}+\mathbf{S}^{(t)} ; \\
w^{(0)}=\frac{1}{16 \pi}\left(\epsilon|U|^{2}+|V|^{2}+|W|^{2}\right) ; \\
S_{y}^{(0)}=-\frac{c}{8 \pi} \operatorname{Re}\left(U^{*} W\right) ; \quad S_{z}^{(0)}=\frac{c}{8 \pi} \operatorname{Re}\left(U^{*} V\right), \quad S_{x}^{(0)}=0 .
\end{gathered}
$$

Функции $V$ и $W$ связаны с $U$ соотношениями (см. также [12])

$$
V(z)=-i U^{\prime}(z) / k, \quad W(z)=-U(z) n_{0, y},
$$

поэтому решение задачи сводится к нахождению функции $U(z)$; здесь далее штрих обозначает производную по $z$. В частности, вне и внутри интервала $[0, d]$ исходное (трехмерное) волновое уравнение для $E_{x}$ сводится, соответственно, к одномерным уравнениям для $U(z)$,

$$
U^{\prime \prime}+k^{2} n_{0, z}^{2} U=0, \quad U^{\prime \prime}+k^{2}\left(n^{2}-n_{0, y}^{2}\right) U=0
$$

где $n_{0, z}=n_{0} \cos \theta$. На границах раздела $z=0$ и $z=d$ функция $U(z)$ и её первая производная $U^{\prime}(z)$ должны быть непрерывными. Это следует из 
граничных условий для тангенциальных проекций $E_{x}, H_{y}$ и ортогональной проекции $H_{z}$, а также из соотношений $(2)$.

Учитывая соотношения $(1)$ для $w^{(0)}, S_{y}^{(0)}$ и $S_{z}^{(0)}$, получаем

$$
\begin{gathered}
w^{(0)}(z)=\frac{1}{16 \pi}\left[\left(n^{2}+n_{0, y}^{2}\right)|U(z)|^{2}+\frac{\left|U^{\prime}(z)\right|^{2}}{k^{2}}\right], \\
S_{y}^{(0)}=\frac{c n_{0, y}}{8 \pi}|U|^{2}, \quad S_{z}^{(0)}=\frac{c}{8 \pi k} \operatorname{Im}\left(U^{*} U^{\prime}\right)=\frac{c n_{0, z}}{8 \pi} T .
\end{gathered}
$$

Отсюда, в частности, следует, что в случае плоской монохроматической световой волны помимо обычного закона сохранения энергии для электромагнитного поля действует еще один закон сохранения -

$$
S_{z}^{(0)}=\frac{c n_{0, z}}{8 \pi} T=\text { const; }
$$

$S_{z}^{(0)}$ - аналог плотности потока вероятности в одномерной квантовой стационарной задаче рассеяния.

Запишем решения уравнений (3). В области $z \leqslant 0$ имеются падающая и отраженная волны

$$
U(z)=\exp \left(i k n_{0, z} z\right)+b_{\text {out }}(k) \exp \left(-i k n_{0, z} z\right) ;
$$

в области $z>d$ есть только прошедшая волна

$$
U(z)=a_{\text {out }}(k) \exp \left[i k n_{0, z}(z-d)\right]
$$

внутри слоя, для $0 \leqslant z \leqslant d$,

$$
\begin{gathered}
U(z)=A_{f} G_{1}\left(x-z_{c} ; k\right)+B_{f} G_{2}\left(z-z_{c} ; k\right) ; \\
a_{\text {out }}=\frac{1}{2}\left(\frac{Q}{Q^{*}}-\frac{P}{P^{*}}\right), \quad b_{\text {out }}=-\frac{1}{2}\left(\frac{Q}{Q^{*}}+\frac{P}{P^{*}}\right) ; \\
A_{f}=-\frac{P^{*}}{\kappa} a_{\text {out }}, \quad B_{f}=\frac{Q^{*}}{\kappa} a_{\text {out }} ; \quad z_{c}=\frac{d}{2} ; \\
Q=\left[G_{1}^{\prime}(z)+i k G_{1}(z)\right]_{z=z_{c}} ; \quad P=\left[G_{2}^{\prime}(z)+i k G_{2}(z)\right]_{z=z_{c}} ;
\end{gathered}
$$

если $n_{0, y} \leqslant n$, то

$$
G_{1}=\sin (\kappa z), \quad G_{2}=\cos (\kappa z), \quad \kappa=k \sqrt{n^{2}-n_{0, y}^{2}} ;
$$

в случае НПВО, то есть, когда $n_{0, y}>n$

$$
G_{1}=\sinh (\tilde{\kappa} z), \quad G_{2}=\cosh (\tilde{\kappa} z), \quad \tilde{\kappa}=k \sqrt{n_{0, y}^{2}-n^{2}} .
$$

Здесь $\left|a_{\text {out }}\right|^{2}=T$ - коэффициент прохождения, $\left|b_{\text {out }}\right|^{2}=R-$ коэффициент отражения; $T+R=1$.

2. Рассеивающаяся ТЕ волна как суперпозиция проходящей и отражающейся компонент. Как и в квантовом случае [9-11], для любого $k$ существует 
единственная пара функций $U_{t r}(z)$ и $U_{r e f}(z)$, которые удовлетворяют уравнению

$$
U_{t r}(z)+U_{\text {ref }}(z)=U(z)
$$

а также обладают следующими свойствами: (а) каждая из этих функций, в отличие от $U(z)$, имеет одну уходящую волну и одну падающую волну; (б) уходящая волна в $U_{t r}(z)$ совпадает с прошедшей волной (см. (5)), а уходящая волна в $U_{r e f}(z)$ совпадает с отраженной волной (см. (4)); (c) падающая волна в каждой из этих двух функций каузально связана на плоскости $z=z_{c}$ с соответствующей уходящей волной - комплекснозначные функции $U_{t r}(z)$ и $U_{r e f}(z)$, а также соответствующие плотности потока энергии непрерывны на этой плоскости (но первая производная каждой из этих функций разрывна). Запишем решения для всех трёх областей:

$$
\begin{gathered}
U_{t r}(z)=A_{t r}^{i n} e^{i k n_{0, z} z}, \quad U_{r e f}(z)=A_{r e f}^{i n} e^{i k n_{0, z} z}+b_{\text {out }}(k) e^{-i k n_{0, z} z} \\
U_{t r}(z)=D_{t r} G_{1}\left(z-z_{c} ; k\right)+B_{f} G_{2}\left(z-z_{c} ; k\right), U_{\text {ref }}(z)=D_{r e f} G_{1}\left(z-z_{c} ; k\right) ; \\
U_{t r}(z) \equiv U(z), \quad U_{r e f} \equiv 0 \\
D_{t r}=-\frac{P Q^{*}}{P^{*} Q} A_{f}, \quad D_{r e f}=\frac{1}{\kappa}\left(P A_{r e f}^{\text {in }}+P^{*} b_{\text {out }}\right) \\
A_{\text {tr }}^{\text {in }}=a_{\text {out }}\left(a_{\text {out }}^{*}-b_{\text {out }}^{*}\right), \quad A_{\text {ref }}^{\text {in }}=b_{\text {out }}^{*}\left(a_{\text {out }}+b_{\text {out }}\right)
\end{gathered}
$$

Выражения (7) соответствуют области $z \leqslant 0$, выражения (8)- области $0 \leqslant z \leqslant z_{c}$, а выражения $(9)$ - области $z>z_{c}$.

Заметим, что, во-первых, $A_{t r}^{\text {in }}+A_{r e f}^{\text {in }}=1$ и $\left|A_{t r}^{i n}\right|^{2}+\left|A_{r e f}^{i n}\right|^{2}=1$; во-вторых, отражающаяся ТЕ компонента не пересекает плоскость $z=z_{c}$; в-третьих, несмотря на тот факт, что производная $U_{t r}^{\prime}(z)$ разрывна на плоскости $z=z_{c}$, ее абсолютное значение $\left|U_{t r}^{\prime}(z)\right|$ непрерывно, поскольку

$$
\left|U_{t r}\left(z_{c}-z\right)\right|=\left|U_{t r}\left(z-z_{c}\right)\right|, \quad\left|U_{t r}^{\prime}\left(z_{c}-z\right)\right|=\left|U_{t r}^{\prime}\left(z-z_{c}\right)\right| .
$$

Эти соотношения следуют из равенства $\operatorname{Re}\left(D_{t r} B_{f}^{*}\right)=\operatorname{Re}\left(A_{f} B_{f}^{*}\right)$, которое следует, в свою очередь, из выражений (6) и (10).

Все это означает, что для проходящей ТЕ компоненты (вещественные) проекции $E_{x}^{t r}$ и $H_{y}^{t r}$, а также плотность энергии $w^{t r}$ и вектор Умова-Пойнтинга $\mathbf{S}^{t r}$ непрерывны на плоскости $z=z_{c}$ :

$$
\begin{gathered}
\mathbf{S}^{t r}=\left(0, S_{y}^{t r}, S_{z}^{t r}\right) ; \quad S_{y}^{t r}=\frac{c n_{0, y}}{8 \pi}\left|U_{t r}\right|^{2}, \quad S_{z}^{t r}=S_{z}^{(0)} \\
w^{t r}(z)=\frac{1}{16 \pi}\left[\left(n^{2}+n_{0, y}^{2}\right)\left|U_{t r}(z)\right|^{2}+\frac{\left|U_{t r}^{\prime}(z)\right|^{2}}{k^{2}}\right] .
\end{gathered}
$$

Но (вещественная) проекция $H_{z}^{t r}$ терпит разрыв на этой плоскости.

Итак, рассеивающаяся световая ТЕ волна единственным образом разлагается в сумму двух каузально распространяющихся ТЕ компонент - проходящей через слой и отражающейся от него. И теперь, когда каждая компонента определена во всех пространственных областях, мы можем приступить к изучению временных аспектов (подпроцесса) туннелирования.

3. О скорости переноса энергии световой волны через слой. Поскольку рассматривается стационарная задача рассеяния, скорость $\mathbf{v}^{\operatorname{tr}}(z)\left(\mathbf{v}^{t r}=\right.$ $\left.=\left(0, v_{y}^{t r}, v_{z}^{t r}\right)\right)$ в точке $z$ для проходящей через слой ТЕ компоненты вводится 
здесь как отношение вектора Умова-Пойтнига к плотности энергии (см. выражения (12)): $\mathbf{v}^{t r}(z)=\mathbf{S}^{t r}(z) / w^{t r}(z) ; \mathbf{v}^{t r}(z)$ - энергетическая скорость. Таким образом, время прохождения световой волны через слой может быть определено следующим образом:

$$
\tau_{D}^{t r}=\int_{0}^{d} \frac{d z}{v_{z}^{t r}(z)}=\frac{1}{S_{z}^{t r}} \int_{0}^{d} w^{t r}(z) d z .
$$

Эту величину будем называть временем пребывания для прохождения (transmission dwell time).

В стандартном подходе скорость света внутри слоя определяется либо как $\mathbf{v}_{D}(z)=\mathbf{S}_{i n c}^{(0)} / w^{(0)}(z)$, где $\mathbf{S}_{i n c}^{(0)}$ - плотность потока энергии падающей ТЕ волны, либо как $\mathbf{v}_{g S}=\mathbf{S}^{(0)} / w^{(0)}(z)$. Первому определению скорости соответствует так называемое буттикеровское время пребывания $\tau_{D}$ :

$$
\tau_{D}=\frac{1}{I_{\text {inc }}} \int_{0}^{d} w^{(0)}(z) d z, \quad I_{\text {inc }}=\frac{c n_{0, z}}{8 \pi},
$$

а второму - время туннелирования $t_{g S}$ (см., например, [13] и ссылки в ней):

$$
t_{g S}=\frac{1}{S_{z}^{(0)}} \int_{0}^{d} w^{(0)}(z) d z
$$

Заметим, что $\tau_{D}$, как и вигнеровское время туннелирования, выходит на насыщение в режиме НПВО с ростом ширины слоя. В то же время $t_{g S}$, отличающееся от $\tau_{D}$ нормировкой, при этом растет экспоненциально, то есть согласуется со специальной теорией относительности. Однако это мало что меняет, поскольку время $t_{g S}$, как и $\tau_{D}$, определено через $w^{(0)}(z)$ и, следовательно, обе величины не имеют никакого отношения к туннелированию.

Детальный анализ скорости переноса энергии для световой компоненты, которая проходит через слой, выполнялся на примере стекла и вакуума. Заметим, что $v^{t r}(z)=c / n_{0}$ вне интервала $[0, d]$; здесь $v^{t r}=\left|\mathbf{v}^{t r}\right|$. Внутри этого интервала функция $v^{t r}(z)$ изменяется, однако ее значения нигде не превышают предельной скорости $c$. Для $n>n_{0}$ скорость $v^{t r}(z)$ имеет максимумы в тех точках $z$, где $\sin (\kappa z)=0$. Это множество точек всегда непустое, поскольку оно обязательно содержит граничные точки $z=0$ и $z=d$. В любой точке этого множества

$$
v^{t r}=v_{\max }^{(1)}=\frac{c}{n} \cdot \frac{2 n_{0} n}{n_{0}^{2}+n^{2}}<\frac{c}{n} .
$$

Для $n_{0}>n \geqslant n_{0, y}$ скорость $v^{t r}$ имеет максимумы в тех точках $z$, где $\cos (\kappa z)=0$ :

$$
v^{t r}=v_{\max }^{(2)}=\frac{c}{n} \cdot \frac{2 k^{2} n_{0, z} n \sqrt{\kappa^{4}+k^{4} n_{0, y}^{2} n_{0, z}^{2}}}{k^{4} n_{0, z}^{2} n^{2}+\left(\kappa^{4}+k^{4} n_{0, y}^{2} n_{0, z}^{2}\right)} \leqslant \frac{c}{n} .
$$

Если $n=n_{0, y}$, то функция $v^{t r}(z)$ принимает максимальное значение $c / n$ в точке $z=z_{c}$. Это единственный случай, когда скорость света в среде, заполняющей слой $[0, d]$, достигает скорости света в данной среде, заполняющей все бесконечное пространство. 
В режиме НПВО максимальное значение скорости $v^{\operatorname{tr}}\left(z_{c}\right)$ убывает, когда угол $\theta$ растет; если $\theta$ превышает некоторое критическое значение, функция $v^{\operatorname{tr}}(z)$ принимает максимальное значение $v_{\max }^{(1)}$ в граничных точках $z=0$ и $z=d$. Что касается точки $z_{c}$, в пределе $\theta \rightarrow 90^{\circ}, v^{t r}\left(z_{c}\right)=c / n_{0}$.

Заметим, что поведение функций $\mathbf{v}^{t r}(z)$ и $\mathbf{v}_{g S}(z)$ для рассматриваемой слоистой структуры различается качественно. Благодаря соотношениям (11) справедливо равенство $\mathbf{v}^{t r}\left(z_{c}-z\right)=\mathbf{v}^{t r}\left(z-z_{c}\right)$. В то же время «скорость туннелирования» $\mathbf{v}_{g S}(z)$, на основе которой определено «групповое время туннелирования» (13), не удовлетворяет этому требованию.

Однако это требование должсно выполняться для исследуемой структуры, поскольку и фоновая среда, и среда внутри слоя с плоско-параллельными границами однородны. Все зеркально симметричные точки в такой структуре физически эквивалентны. Поэтому скорость туннелирования должна быть четной функцией переменной $z-z_{c}$. Отражающаяся компонента, которая существует только в левой половине интервала $[0, d]$, не должна влиять на значение скорости туннелирования в этой области.

Заключение. Предложена новая модель рассеяния плоской ТЕ-поляризованной световой волны на однородном диэлектрическом слое, которая дает раздельное описание подпроцессов прохождения и отражения на всех этапах рассеяния. Показано, что рассеивающаяся ТЕ волна может быть единственным образом представлена в виде суперпозиции двух ТЕ волн, одна из которых описывает прохождение, а другая - отражение. Согласно этой модели проходящая через однородный слой компонента световой волны должна проходить через левую и правую половины этого слоя за одинаковое время. При этом время прохождения через весь слой должно расти экспоненциально в режиме НПВО с ростом ширины слоя, что указывает на отсутствие эффекта Хартмана. В рамках новой модели скорость туннелирования в зеркальносимметричных точках исследуемой структуры одинакова, а отражающаяся TE компонента не пересекает плоскость симметрии структуры.

Предполагается, что динамику каждого из подпроцессов можно исследовать экспериментально на основе эффекта Фарадея, если (инфинитезимальное) магнитное поле включать в том или ином интервале на оси $O Z$. Для этого планируется разработать соответствую математическую модель.

Работа выполнена при частичном финансировании Программы поддержки ведущих научных школ Российской федерации (проект № 224.2012.2).

\section{БИБЛИОГРАФИЧЕСКИЙ СПИСОК}

1. T. E. Hartman, "Tunneling of a Wave Packet"// J. Appl. Phys., 1962. Vol.33, no. 12. Pp. 3427-3433.

2. J. Jakiel, V. S. Olkhovsky, E. Recami, "On superluminal motions in photon and particle tunnellings" // Phys. Lett. A, 1998. Vol. 248, no. 2-4. Pp. 156-160.

3. G. Nimtz, "Tunneling Confronts Special Relativity" // Found. Phys., 2011. Vol.41, no. 7. Pp. 1193-1199, arXiv: 003.3944 [quant-ph].

4. D. J. Papoular, P. Clade, S. V. Polyakov, C. F. McCormick, A. L. Migdall, P. D. Lett, "Measuring optical tunneling times using a Hong-Ou-Mandel interferometer" // Optics Express, 2008. Vol. 16, no. 20. Pp. 16005-16012.

5. N. Borjemscaia, S. V. Polyakov, P. D. Lett, A. Migdall, "Single-photon propagation through dielectric bandgaps" // Optics Express, 2009. Vol.18, no. 3. Pp. 2279-2286.

6. N. Brunner, V. Scarani, M. Wegmuller, M. Legrre, N. Gisin, "Direct measurement of 
superluminal group velocity and signal velocity in an optical fiber" // Phys. Rev. Lett., 2004. Vol. 93, no. 20, 203902. 4 pp., arXiv: quant-ph/0407155.

7. R. Y. Chiao, J. Boyce, M. W. Mitchell, "Superluminality and parelectricity: The ammonia maser revisited" // Appl. Phys. B, 1995. Vol.60, no. 2-3. Pp. 259-265.

8. M. Büttiker, R. Landauer, "Traversal time for tunneling"// Phys. Rev. Lett., 1982. Vol.49, no. 23. Pp. 1739-1742.

9. Н. Л. Чуприков, "Новый взгляд на квантовый процесс туннелирования: Волновые функции для прохождения и отражения" // Изв. вузов. Физика, 2006. Т. 49, № 2. С. 39; англ. пер.: N. L. Chuprikov, "New approach to the quantum tunnelling process: wave functions for transmission and reflection" // Russ. Phys. J., 2006. Vol. 49, no. 2. Pp. 119-126.

10. N. L. Chuprikov, "On a new mathematical model of tunnelling"// Vestn. Samar. Gos. Univ. Estestvennonauchn. Ser., 2008. no. 8/1(67). Pp. 625-633.

11. N. L. Chuprikov, "From a 1D Completed Scattering and Double Slit Diffraction to the Quantum-Classical Problem for Isolated Systems"// Found. Phys., 2011. Vol.41, no.9. Pp. 1502-1520.

12. M. Born, E. Wolf, Principles of Optics: Electromagnetic Theory of Propagation, Interference and Diffraction of Light. New York: Cambridge U. P., 1999 [1959]. 952 рр.; русск. пер.: М. Борн, Э. Волъф, Основы оптики. М.: Наука, 1970. 856 с.

13. А. Б. Шварцбург, "Туннелирование электромагнитных волн - парадоксы и перспективы" // УФН, 2007. Т. 177, №1. С. 43-58; англ. пер.: A. B. Shvartsburg, "Tunneling of electromagnetic waves: paradoxes and prospects" // Phys. Usp., 2007. Vol.50, no. 1. Pp. 37-51.

Поступила в редакцию 01/XI/2012;

в окончательном варианте - 17/III/2013.

MSC: 81U05, 35L05; 81905, 81P05, 81P15, 83C10

\section{ON THE HARTMAN EFFECT AND VELOCITY OF PROPAGATING THE ELECTROMAGNETIC WAVE IN THE TUNNELING PROCESS}

\section{N. L. Chuprikov}

Tomsk State Pedagogical University,

60, Kievskaya st., Tomsk, 634061, Russia.

E-mail: chnl@tspu.edu.ru

A new approach to the problem of scattering the plane electromagnetic TE wave on a homogeneous dielectric layer is presented, which does not predict, unlike the standard model, the Hartman effect for the tunneling time in the case of scattering the TE wave in the regime of a frustrated total internal refection (FTIR). The basic idea of this approach is that a correct definition of the tunneling velocity and time is possible if only the dynamics of both its subprocesses - transmission and reflection - is known at all stages of the scattering process investigated. It is shown that the Wigner (group) tunneling time was introduced without taking into account of this requirement, and, as a consequence, both this characteristic itself and the associated with it Hartman effect have no relation to transferring the light energy through the layer. The dwell transmission time, which is directly related to it, does not lead to the Hartman effect.

Key words: group velocity, energy velocity, tunneling time, transmission, reflection.

Original article submitted 01/XI/2012; revision submitted $17 / \mathrm{III} / 2013$.

Nikolay L. Chuprikov (Dr. Sci. (Phys. \& Math.)), Professor, Dept. of Theoretical Physics. 